18 Perkins R, Rinaldi M. A Guide to Planning Your Own Recovery. South West London and St George's Mental Health NHS Trust, 2007.

19 Corrigan P, Muser K, Bond G, Drake R, Soloman P. Peer services and supports. In Principles and Practice of Psychiatric Rehabilitation: 346-58. Guilford Press, 2008

20 Department of Health. National Service Framework for Mental Health: Modern Standards and Service Models. Department of Health, 1999.

21 Hope R. The Ten Essential Shared Capabilities: A Framework for the Whole of the Mental Health Workforce. Department of Health, 2004

22 Slade M. Personal Recovery and Mental Illness: A Guide for Mental Health Professionals. Cambridge University Press, 2009: 231-3.
23 Shepherd G, Boardman J, Slade M. Making Recovery a Reality. Sainsbury Centre for Mental Health, 2008.

24 Royal College of Psychiatrists. A Competency Based Curriculum for Specialist Training in Psychiatry. Royal College of Psychiatrists, 2009 (http://www.rcpsych.ac.uk/training/curriculum2009.aspx).

25 Roberts G, Hollins S. Recovery: our common purpose? Adv Psychiatr Treat 2007; 13: 397-9.

26 Roberts G, Dorkins E, Wooldridge J, Hewis E. Detained - what's my choice? Part 1: Discussion. Adv Psychiatr Treat 2008; 14: 172-80.

27 Copeland M, Mead S. WRAP and Peer Support: A Guide to Individual, Group and Program Development. Peach Press, 2003.

\title{
The Mental Capacity Act 2005: review of mental capacity assessment in people with proximal femoral fracture
}

\author{
P. Guyver, ${ }^{1}$ P. Hindle, ${ }^{1}$ J. Harrison, ${ }^{2}$ N. Jain, ${ }^{1}$ M. Brinsden ${ }^{1}$
}

The Psychiatrist (2010), 34, 284-286, doi: 10.1192/pb.bp.109.026757

${ }^{1}$ Derriford Hospital, Plymouth; ${ }^{2}$ HMNB Portsmouth

Correspondence to J. Harrison (james.harrison650@mod.uk)

\begin{abstract}
Aims and method To ascertain whether patients with proximal femoral fractures were being correctly assessed in line with the Mental Capacity Act 2005 . Fifty people admitted with proximal femoral fractures were audited to assess whether they had given consent to treatment in accordance with the Act. A Mental Capacity Act 2005 guidance and assessment form was then introduced accompanied by staff training. $A$ re-audit was undertaken to assess the impact.
\end{abstract}

Results The initial audit showed that only one person (2\%) had been properly assessed. The re-audit demonstrated that the use of the Mental Capacity Act 2005 assessment form ensured correct assessment.

Clinical implications Our findings suggest the form is a useful tool in the documentation and assessment of an individual's capacity under the Mental Capacity Act.

Declaration of interest None.
It is now over a year since the implementation of the Mental Capacity Act 2005 in England and Wales. This new legislation and its impact on clinicians has been described elsewhere, ${ }^{1}$ however one of the key requirements of the Act is that: 'The person who assesses an individual's capacity to make a decision will usually be the person who is directly concerned with the individual at the time the decision needs to be made'. ${ }^{2}$ The act of giving consent to any procedure performed in a healthcare setting is now subject to the Mental Capacity Act and its requirements, and a legal framework exists to ensure that both the decision maker and the patient have rights and responsibilities.

The direct responsibility of the clinician performing a procedure has also subtly changed under the Act; now, this decision maker has to take responsibility for the decision even if he seeks advice from another professional (such as a psychiatrist) when assessing the patient's capacity. This change therefore makes it a requirement for all clinicians to be able to assess, describe and document capacity with regard to the specific procedures. When confronted with an individual requiring a surgical procedure for instance, the Mental Capacity Act requires that the consenting doctor fully assesses the person before deciding upon a specific course of treatment. This assessment process is clearly described within the Act and essentially consists of two stages.

1 Does the person have an impairment of, or a disturbance in the functioning of their mind or brain? If the answer to this is no then the patient is assumed under the Act not to lack capacity, and so a procedure of informed consent can be instigated. The Code of Practice ${ }^{2}$ lists examples of conditions that may lead to this impairment 
or disturbance of function (Box 1). It is important to note that the Act requires 'that it is more likely than not that the person lacks capacity to make a particular decision', ${ }^{2}$ in order to make the claim that the individual lacks capacity.

2 Does the impairment or disturbance mean that the person is unable to make a specific decision when they need to? The Act goes on to say that the individual's impairment or disturbance must affect their ability to make a specific decision when they need to. A legal test is described in the Act that must be utilised to decide whether a person is unable to make a decision (Box 2).

Formally assessing these components of the Act will require a degree of training on the part of non-specialist clinicians, both in terms of the assessment procedure and its documentation.

It is clear then that vulnerable individuals undergoing surgical procedures need adequate assessment of their capacity before consent can be considered valid. If the clinician obtaining consent from the person feels that they lack capacity in accordance with the Act, then they should proceed with treating the individual in their 'best interests'. The implication of this aspect of the Act is that the clinician making the decision should try to use a number of different sources of information to ascertain what is in the persons' best interest. These include obtaining the lead clinician's opinion, discussing the options with interested parties such as the next of kin and those with power of attorney, the review of any advanced directives and considering consultation with an Independent Mental Capacity Advocate (IMCA). If there is no one other than the clinician to act on the patient's behalf then consultation with an IMCA is recommended. This is not always practical and decisions may need to be made without consultation because of time constraints and the availability of an advocate. It should, however, always be considered and accessed where practicable.

Over the past 30 years there has been a significant amount of work conducted into the assessment of capacity when considering consent issues. Instruments have been designed to enable the accurate testing of capacity in specific groups. ${ }^{3}$ However, these instruments relate primarily to research into competency rather than the

Box 1 Examples of an impairment of, or disturbance in the functioning of the mind or brain

- Conditions associated with some forms of mental illness

- Dementia

- Significant intellectual disabilities

- The long-term effects of brain damage

- Physical or mental conditions that cause confusion, drowsiness or loss of consciousness

- Delirium

- Concussion following a head injury

- The symptoms of alcohol or drug use

Taken from the Mental CapacityAct Code of Practice (Section 4.12). ${ }^{2}$

\section{Box 2 Impairment}

A person is unable to make a decision if they cannot:

- understand information about the decision to be made (the Act calls this 'relevant information');

- retain that information in their mind;

- use or weigh that information as part of the decision-making process; or

- communicate their decision (by talking, using sign language or any other means).

Taken from Mental CapacityAct 2005 Code of Practice (Section 4.14). ${ }^{2}$

day-to-day clinical assessment that we aimed to address in this audit and that is legislated for in the Mental Capacity Act.

Our audit was undertaken to assess the process of assessment of mental capacity with regard to consent among people with proximal femoral fractures. Following the initial audit, the orthopaedic department introduced a simple form to guide the assessment of capacity in this group of patients. A re-audit was then completed to elicit the utility of the form in capacity assessment.

\section{Method}

A prospective audit of 50 consecutive patients admitted to the orthopaedic department of Derriford Hospital, Plymouth, with proximal femoral fractures was undertaken. The consent procedure and mental capacity documentation were reviewed along with the notes recording the medical history and clinical assessment. In the absence of a data-set in the medical records, an assessment was assumed not to have been undertaken, commensurate with General Medical Council guidelines. ${ }^{4}$

From the clinical records an assessment was made as to whether stage 1 of the capacity test was fulfilled, i.e. the individual had 'an impairment of the mind or brain, or some sort of disturbance that affects the way their mind or brain works'. ${ }^{2}$ Information used to guide this assessment included the presence of other diagnoses at the time of consent, as well as documentation of the physical and mental state at the time. This allowed the reviewers to make decisions about whether the person exhibited impaired capacity. If impairment was not found at stage 1 then the individual was assumed to have been able to give voluntary consent. In all other instances they were deemed to have incapacity to consent. In accordance with the Mental Capacity Act Code of Practice guidance, incapacity to consent was deemed to be present if it was more likely than not that the person lacked capacity.

The notes of individuals in the voluntary consent group were investigated further to see whether any of them had documented conditions, assessments or written statements that would indicate that they had failed stage 1 of the capacity test and therefore that a formal assessment of capacity should have been performed.

The notes of people in the incapacity to consent group were reviewed to see whether an assessment of capacity in 
line with stage 2 of the capacity test had taken place. In addition, any documentation of the lead clinician's opinion, advanced decisions, consultation with interested parties (i.e. next of kin) and/or an IMCA were also highlighted.

The results from both the voluntary consent and the incapacity to consent groups were then collated to assess whether people were being assessed and consented in accordance with the Act. A combined Mental Capacity Act assessment and guidance form was devised to improve capacity assessment. A month after introduction of the form and completion of staff training the audit was repeated.

\section{Results}

The original audit of 50 patients showed 34 with voluntary consent and 16 with incapacity to consent. In the voluntary consent group, only one person (3\%) had had their capacity correctly assessed in accordance with all aspects of the new Act. Of the 34, 8 (24\%) had a documented condition that might impair their capacity and 2 individuals had a minimental test score indicative of mild cognitive impairment, but no further assessment of capacity was carried out. These conditions included urinary tract infections, head injury, confusion, cerebrovascular accidents with mild cognitive impairment and loss of memory.

A total of 15 of the 16 incapacity to consent group had a documented condition that might indicate they lacked capacity to consent. Unfortunately, one person had no documented reason as to why they were unable to give voluntary consent. Of the 15, 8 had documented concerns about their capacity; 11 had the lead clinician's opinion on their treatment; 1 had a review of advanced directives; 10 had had discussion with interested parties, but no one had consulted an IMCA.

The re-audit identified 33 with voluntary consent and 12 with incapacity to consent. Five sets of notes were not available for review leaving a total of 45 . In the voluntary consent group, 18 had the form filled out; 11 had the form in the notes but it had not been filled out; and in 4 cases the form was missing. In the incapacity to consent group, 10 of the 12 had a form completed in full. All of the people who had the form filled out (28/45 (62\%)) had been assessed properly in accordance with the Mental Capacity Act Code of Practice (100\% compliance).

\section{Discussion}

Initial audit revealed that $97 \%$ of people giving voluntary consent had no documentation of mental capacity assessment. Had an assessment been undertaken then $24 \%$ of these individuals would have been identified as having a condition that may affect capacity to consent. In the group of people identified as being unable to give voluntary consent only one had been assessed in line with the Mental Capacity Act Code of Practice. In one case there was no documentary evidence as to why the person was unable to give voluntary consent, which may constitute a breach of statutory law. Despite training, the re-audit showed compliance with the assessment form was moderate at $62 \%$, but in those cases in which a form was completed there was $100 \%$ compliance with the Mental Capacity Act Code of Practice.

We propose that the assessment form may be best applied in a targeted fashion, aimed at individuals identified by the multidisciplinary team as having issues in the area of mental capacity and their ability to give voluntary consent.

In summary, the Act empowers individuals (patients), giving them an inherent right to voluntary consent as well as providing a legal framework for those making decisions (health professionals) on behalf of individuals who lack capacity. Voluntary (type 1) consent taken from an individual without capacity may constitute battery and best interests (type 4) consent taken on behalf of an individual with capacity contravenes statutory law in the form of the Mental Capacity Act. Adequate assessment of mental capacity is therefore mandatory when obtaining consent. This study shows that a mental capacity assessment form greatly improves compliance with the Mental Capacity Act Code of Practice.

\section{About the authors}

P. Guyver and P. Hindle work in the Department of Trauma and Orthopaedics at Derriford Hospital, Plymouth Hospitals NHS Trust, Devon. J. Harrison works in the Department of Community Mental Health, HMNB Portsmouth, Hampshire. N. Jain and $\mathbf{M}$. Brinsden work in the Department of Trauma and Orthopaedics at Derriford Hospital, Plymouth Hospitals NHS Trust, Devon, UK.

\section{References}

1 Ashir M, Sahib L. Assessment of mental capacity. Psychiatr Bull 2007; 31: 436

2 Department for Constitutional Affairs. Mental Capacity Act 2005 Code of Practice. TSO (The Stationery Office), 2007.

3 Grisso T, Applebaum P, Mulvey E, Fletcher K. The MacArthur Treatment Competence Study II: measures of abilities related to competence to consent to treatment. Law Hum Behav 1995; 19: 127-48.

4 General Medical Council. Good Medical Practice. GMC, 2006. 